\title{
A GLOBAL HIERARCHICAL AND EQUAL-AREA SHPERE/ ELLIPSOID GRID AND ITS GEOMETRY DISTORTION ANALYSIS
}

\author{
Sun Wenbin*, Zhou Changjiang \\ School of Geoscience and Surviving Engineer, China University of Mining and Technology(Beijing), Beijing, China \\ E-mail: swb1996@126.com; 357699853@qq.com
}

\begin{abstract}
KEY WORDS: Global Discrete Gird, Hierarchical Grid, Equal-Area, Snyder Projection, Geometry Distortion Analysis, Sphere Surface
\end{abstract}

\begin{abstract}
:
A spatial data management and analysis frame is required for global problem application. Global Discrete Grid (GDG) has seamless, excellent hierarchy characteristics. GDG has been used for spatial data management, indexing and cartographic generalization. However, most GDGs are unequal-area. To extend GDG application ranges in spatial modelling and statistical analysis, the method for constructing hierarchical and equal-area GDG is discussed in this paper. The detail steps to build GDG based on inscribe polyhedron is presented. The method of transferring polyhedron surface grids onto sphere surface is described. The ratio of max, min length of grid edges and grid angle is acquired. Length ratio converges to1.7 and angle ratio converges to 3.0. The result indicates that there exists difference in length and grid angle and the ratios of them are convergent.
\end{abstract}

\section{INTROUDUCTION}

With the development of requirement of global problems research, multi-scale spatial data is used to analyse global problems, such as global tide simulation, atmospheric modelling and global resources management etc[Titz etc 2004; Bartholdi \& Glodsman 2003; Dutton 1999, 2000; Goodchild 1991]. So a data frame is required to store spatial data and is also considered as basic cells for global problem analysis. Hierarchical characters provide much convenience in multiscale spatial data management because hierarchy data frame and multi-scale structure have homogeneity characteristics [Kiester \& Sahr 2008; Kolar 2004]. Equal-area is another important character in spatial sampling, statistics analysis. Therefore, to construct hierarchical equal-area global grid is very significant for global problem application.

There are many models for global problem analysis. But projection transformation is considered as the base of most of these models. Traditionally, the sphere (ellipsoid) surface is divided into a number of regions to decrease distortion during projecting process and spatial analysis operation of each region is carried out. It is obvious that projection and topology errors are inevitable because sphere surface belongs to manifold surface, which is different with plane Euclidean space [Kimerling etc1999; Ming \& Zhuang 2009; Ming etc 2011]. Direction, length and area metric methods are different in two spaces and the results of computing direction, length, area cannot preserve uniformity synchronously. So, a structureGlobal Discrete Grid (GDG), which is infinite subdivision tessellation on spherical surface and could be used to simulate global scale phenomena if the subdivision is repeated, is introduced in geographic science. GDG is approximate alternative to manage global scale spatial data.

The theories and methods to construct GDG have been studied widely[Gregory etc 2008; Ma etc 2009; Leopardi 2006; Oldham etc 2012; Rosca \& Plonka 2011; Sahr 2003, 2008; Savric etc 2011; Schneider \& Klein 2007; Yuan etc 2010; Vine \& Zhang 2009; White 2000]. There are two main way to subdivide sphere/ellipsoid surface for constructing GDG. The first one is to subdivide sphere/ellipsoid surface into tessellation directly, such as Longitude/latitude grid, constant "area” gird, Seong’s equal-area grid, Song's small circle grid, degenerate quadtree grid, etc[Bjoke 2003, 2004; Seong 2005; Song etc 2000; Zhao etc 2009]. Longitude/latitude grid is one of simplest GDG. Traditionally, equal-interval subdivision scheme is selected to build longitude/latitude gird. This kind of grid has been used in many fields, such as GTOPO30 data management, image data index, etc. But the sizes of equal-interval degree longitude/latitude grids are very different from the equator to the poles and the grids near the poles need to be disposed individually. To decrease area distortion, variable-interval scheme is approached for constant "area" grid[Bjoke 2003, 2004]. In this scheme, small degree interval is used near the equator and large degree interval is implemented near the poles. But area distortion of this kind grid is not completely eliminated and this leads to poor preformation in statistical analysis. To overcome this deficiency, equal-area grids are introduced in some field, such as Seong,s grid and Song's grid etc[Seong 2005; Song etc 2000; Leopardi 2006; Rosca \& Plonka 2011]. Seong's grid can be constructed by measuring equal distance along meridian and parallel direction separately[Seong 2005]. Although most of Seong's girds are equal-area, area of the last cell of each row is not equal with others. What is more, it is very difficult to carry out neighbour query in Seong's scheme because there is no simple and clear neighbour relation among grids. In other words, ignoring neighbour relation to obtain equal-area character as a reward is not perfect. Another equalarea subdivision scheme is given by Song[Song etc 2000]. In this method, each cell is spherical triangle and two vertexes of each cell are connected by using sphere small arcs. Heavy computation is needed during the process of creating those grids because edges of cells are not geodesic. Besides these schemes, degenerate quadtree $\operatorname{grid}(\mathrm{DQG})$ is implemented for DEM visualization by Zhao[Zhao etc 2009]. In this method, sphere surface is subdivided according to quadtree principles firstly; then two cells near the pole are merged into one cell; the same subdivision processes are repeated and DQG grid is approached.

* Corresponding author. The School of Surveying Engineering and Geo-science, China University of Mining \& Technology, Beijing, 100083, China. E-mail addresses: swb1996@126.com . 
DQG provides excellent hierarchy but poor performance in equal-area character. From discussion above, we can find that there is not simple subdivision scheme for constructing hierarchical and equal-area grid on sphere/ellipsoid surface directly.

Another method has also been used widely by subdividing auxiliary object surface into cell and mapping cells onto sphere/ellipsoid surface[White 2000; Dutton 2000; Goodchild etc 1991]. Traditionally, tetrahedron, hexahedron, octahedron, dodecahedron, icosahedrons and truncated polyhedron are selected as auxiliary objects[White 2000;Kiester \& Sahr 2008]. The discrepancy of auxiliary object and sphere surface is not big. Therefore, those GDGs have also been used in many fields. Different auxiliary objects have different shape face, such as triangle, square, pentagon, hexagon, etc. Each face of auxiliary objects is subdivided into four or nine hierarchical cells and those cells are transferred onto sphere surface in different way[White 2000; Rosca \& Plonka 2011; Sahr 2003]. Snyder(1992) gives a projection method to transfer cells of polyhedron surface onto the globes while equal-area characteristic of each cells is preserved. An optimization method about Snyder equal-area projection is presented by Harrison[2011, 2012].

Although equal-area and hierarchical GDGs have been studied, there is no perfect subdivision scheme to acquire equal-area, hierarchical cells on ellipsoid surface. Therefore, the methods to subdivide equal-area cell on sphere surface is discussed and the way to extend sphere to ellipsoid is tried to be discussed in this paper.

\section{EQUAL-AREA SUBDIVISION}

\section{1 the principle of constructing equal-area grid}

Subdivision scheme base on inscribed polyhedron provides approximate shape cells, which have excellent hierarchy characteristic and simple neighbour relation. Octahedron is one of inscribed polyhedron used widely by many researchers. Octahedron is the only inscribed polyhedron, which can be compressed and embed into an ellipsoid, shown as figure1. Therefore, octahedron is selected as subdivision polyhedron in this paper. In figure1, vertexes A and B are opposite pair vertexes of octahedron. To embed octahedron into ellipsoid, the distance of vertexes $A$ and $B$ is decreased. When the distance of $\mathrm{AB}$ is equal to twice length of ellipsoid short axis, compression octahedron can be embed into ellipsoid. Each face of compression octahedron is isosceles triangle and hierarchical subdivision scheme can be carried out.
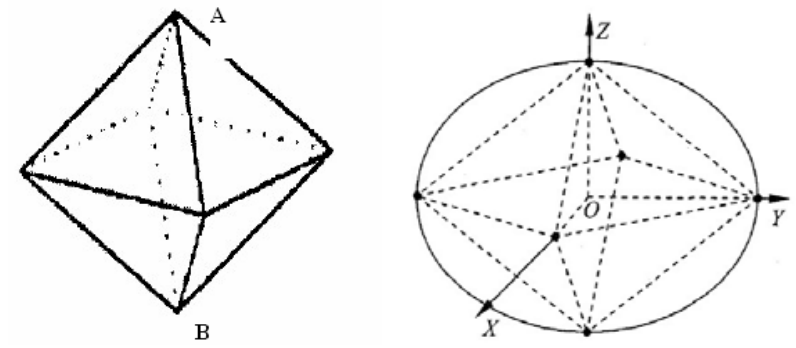

Figure 1 Compression octahedron

To acquire equal-area cell in inscribe polyhedron subdivision scheme, two steps should be done. Firstly, equal-area cell on polyhedron face should be acquired. Secondly, the method transferring cell on polyhedron surface onto sphere/ellipsoid surface should be approached. Acquiring equal-area cell on polyhedron surface is implemented by connecting midpoints of equilateral triangle or isosceles triangle edges. Commonly, equal area projection can be used for transferring polyhedron cells onto sphere/ellipsoid surface. In this paper, two equal area projection methods, azimuthal equal area projection and Snyder projection, are used for getting equal-area cells.

\subsection{Oblique azimuthal equal-area projection}

Oblique azimuthal equal-area projection is selected for transferring polyhedron cells onto sphere/ellipsoid surface because plane is considered as auxiliary object in this projection process. Octahedron should be proportionately enlarged to make polyhedron surface area being equal to sphere/ellipsoid area at first. Then, put octahedron into sphere, shown as figure 2. As octahedron is symmetrical object, one face of octahedron is selected to show the process of subdivision and coordinate construction on octahedron surface. Shown as figure 2, four son-triangles are acquired by connecting midpoint of triangle edges. Gravity center of triangle (one octahedron face) is selected as origin of a plane coordinate system. The coordinates of each vertex of son-triangle can be acquired. After that, two transferring steps (transferring plane coordinates into sphere coordinate; transferring sphere coordinate into geodetic coordinates) are needed to transfer plane coordinate $(x, y)$ on octahedron surface to geodetic coordinate $(\lambda, \varphi)$. This process can be implemented according to formula 1 and 2 .

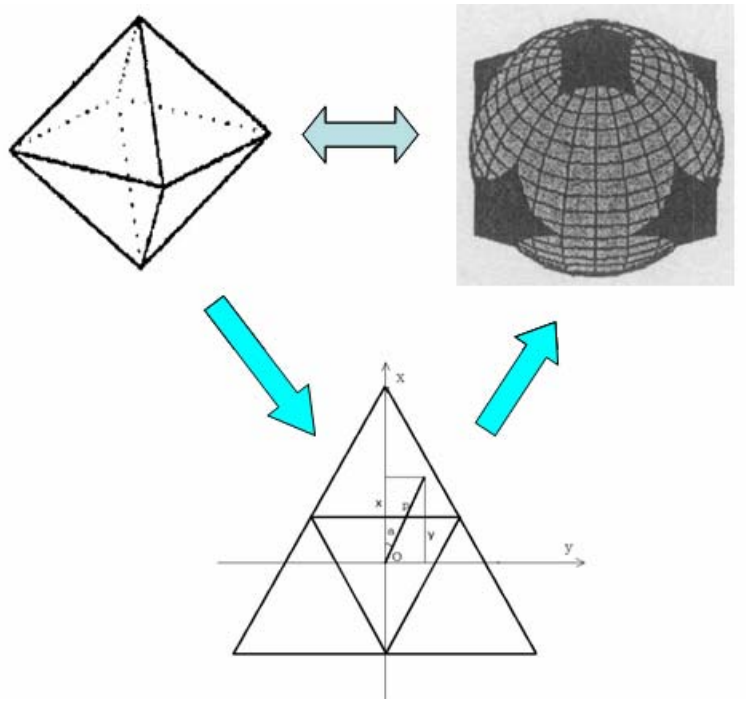

Figure2 The process of coordinate transformation

Plane coordinate to sphere coordinate:

$$
\begin{aligned}
& \alpha=\arctan \frac{y}{x}(x \neq 0) \\
& Z=2 \arcsin \frac{\sqrt{x^{2}+y^{2}}}{2 R}
\end{aligned}
$$

Where $\mathrm{R}$ is sphere radius; (x, y) is plane coordinates; $\alpha, \mathrm{Z}$ is sphere coordinates.

Sphere coordinates to geodetic coordinate:

$$
\begin{aligned}
& \sin \varphi=\sin \varphi_{0} \cos Z+\cos \varphi_{0} \sin Z \cos \alpha \\
& \tan \left(\lambda-\lambda_{0}\right)=\frac{\sin Z \sin \alpha}{\cos Z \operatorname{sos} \varphi_{0}-\sin Z \sin \varphi_{0} \cos \alpha}
\end{aligned}
$$

Where $\lambda, \varphi$ is geodetic coordinates; $\lambda_{0}, \varphi_{0}$ is projection center geodetic coordinates. 
Topics: Global Spatial Grid \& Cloud-based Services

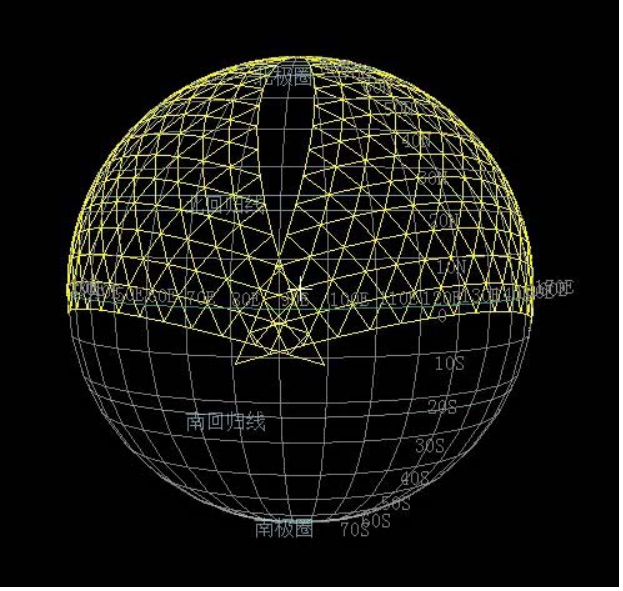

(a)

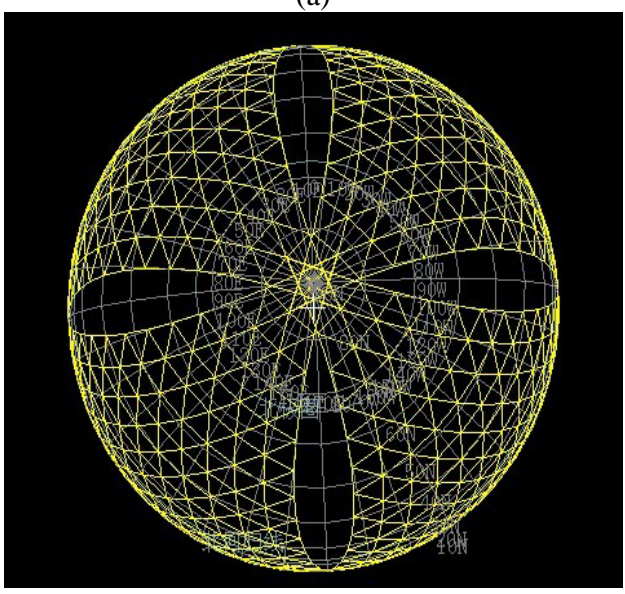

(b)

Figure 3 visualization of azimuthal projection

To learn the result of azimuthal projection, visualization of projected grids is implemented based on the platform of world wind, shown as figure 3. Figure 3.a shows the grids near the equation. Figure 3.b shows the grids near the pole from vertical view. We can find that there exists gaps and overlapping in matching regions of different polygon faces. It is obvious that oblique azimuthal equal-area projection is not perfect selection to transfer polyhedron cells onto sphere surface.

\subsection{Snyder Projection}

Snyder gives an equal-area projection method and relative transferring formulas(3-17), which can be used for transferring polyhedron cells onto sphere surface [Snyder 1992]. In this method, three conditions should be fulfilled. Firstly, surface area of polyhedron is equal to sphere's one; secondly, if azimuths $\alpha$ is known, area of spherical triangle $S_{\triangle A B D}$ is equal to plane triangle $S_{\triangle A^{\prime} B^{\prime} \mathrm{D}}$ area, shown as figure 4; thirdly, differential equations in equal-area transformation process is always correct and the area ratio of non-projection and projected polygon is equal to 1 . Spherical triangle area can be calculated according to formula 3 and 4 based on spherical excess area calculation principle. Plane triangle area is computed according to formula 5-8.
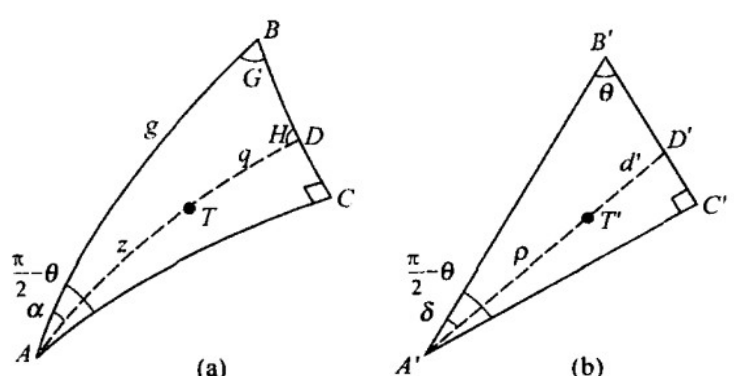

Figure 4 Spherical right triangle and right tirangle(Snyder 1992)

$$
\begin{aligned}
& S_{\triangle A^{\prime} B^{\prime} D^{\prime}}=\frac{1}{2} A^{\prime} B^{\prime} \times A^{\prime} D^{\prime} \times \sin \delta \\
& \tan \frac{g}{R}=\sqrt{2} \\
& A^{\prime} B^{\prime}=R^{\prime} \bullet \tan \frac{g}{R} \\
& A^{\prime} D^{\prime}=A^{\prime} B^{\prime} \bullet \frac{\sin \theta}{\sin (\pi-\delta-\theta)} \\
& R^{\prime}=\sqrt{\frac{\sqrt{3}}{9} \pi \bullet R} \\
& \theta=\pi / 6
\end{aligned}
$$$$
S_{\triangle A B D}=(a+G+H-\pi) \times R^{2} \quad G=\pi / 4
$$$$
H=\cos ^{-1}\left(\sin \alpha \sin G \bullet \cos \frac{g}{R}-\cos \alpha \cos G\right)
$$

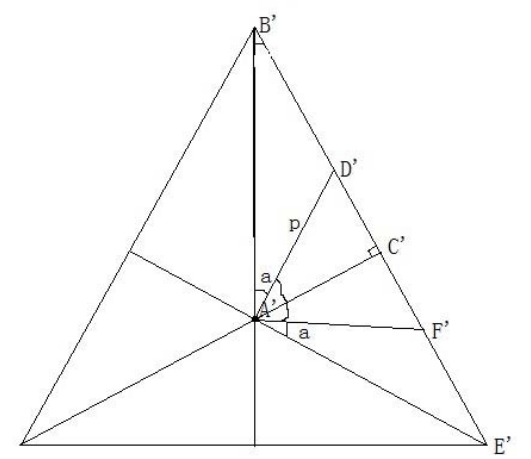

Figure 5 Snyder projection on octahedron surface

To transfer octahedron surface equal-area cell onto sphere surface, back projection process of Snyder projection is implemented. The detailed process is shown as figure 6. Firstly, plane coordinates is set up, shown as figure 2. Then, values of $\rho$ and $\delta$ are calculated according to coordinates (x, y) and formula 9. Next, $S_{\triangle A B D}$ is computed by using formula 10 . After that, sphere coordinate $\alpha$ and $\mathrm{Z}$ is acquired based on formula 11-17. Transferring result shows as figure 8. Figure 8.a shows the grids near the equation. Figure 8.b shows the result of Snyder projection near the pole from vertical view.

$$
\begin{aligned}
& \delta=\tan ^{-1}(y / x) \\
& \rho=\sqrt{x^{2}+y^{2}} \\
& S_{\triangle A B D}=\frac{1}{2}\left(R^{\prime} \tan \frac{g}{R}\right)^{2} \frac{1}{\cot \theta+\cot \delta}
\end{aligned}
$$


International Archives of the Photogrammetry, Remote Sensing and Spatial Information Sciences, Volume XL-4/W2, 2013 ISPRS WebMGS 2013 \& DMGIS 2013, 11 - 12 November 2013, Xuzhou, Jiangsu, China

Topics: Global Spatial Grid \& Cloud-based Services

$$
\begin{aligned}
& F(\alpha)=\frac{S_{\triangle A B D}}{R^{2}}-G-H-\alpha+\pi \\
& H=\cos ^{-1}\left(\sin \alpha \bullet \sin G \bullet \cos \frac{g}{R}-\cos \alpha \cos G\right) \\
& F^{\prime}(\alpha)=\frac{\cos \alpha \sin G \bullet \cos (g / R)+\sin \alpha \cos G}{\sin H}-1 \\
& \Delta \alpha=-\frac{F(\alpha)}{F^{\prime}(\alpha)} \\
& \alpha=\delta+\Delta \alpha \\
& q=R \bullet \tan ^{-1}\left(\frac{\tan (g / R)}{\cos \alpha+\sin \alpha \bullet \cot \theta}\right) \\
& f=\frac{2 \bullet R^{\prime} \bullet \sin \frac{q}{a R}}{d^{\prime}} \\
& d^{\prime}=\frac{R^{\prime} \bullet \tan (g / R) \bullet \sin \theta}{\sin (\delta+\theta)} \\
& Z=2 R \bullet \sin ^{-1}\left(\frac{\rho \bullet f}{2 \bullet R^{\prime}}\right)
\end{aligned}
$$

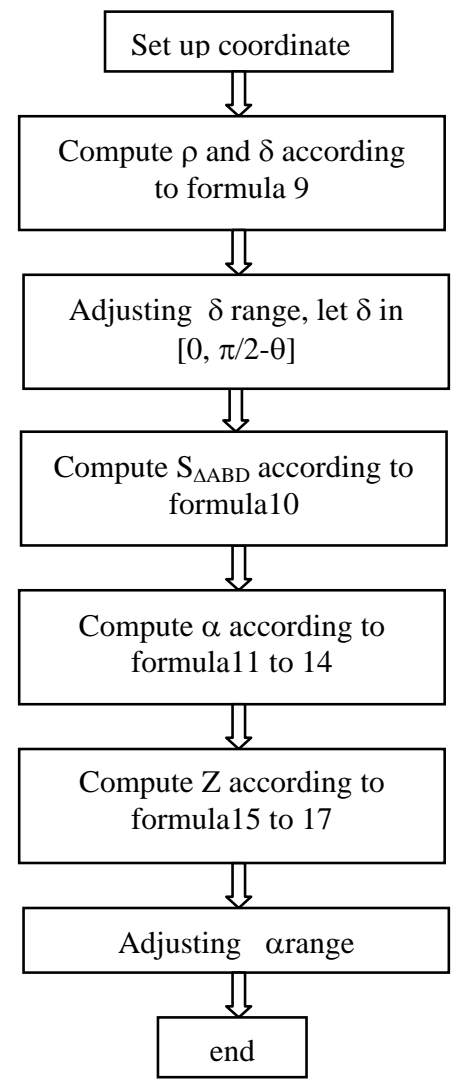

Figure 6 The flow chart of Snyder back projection

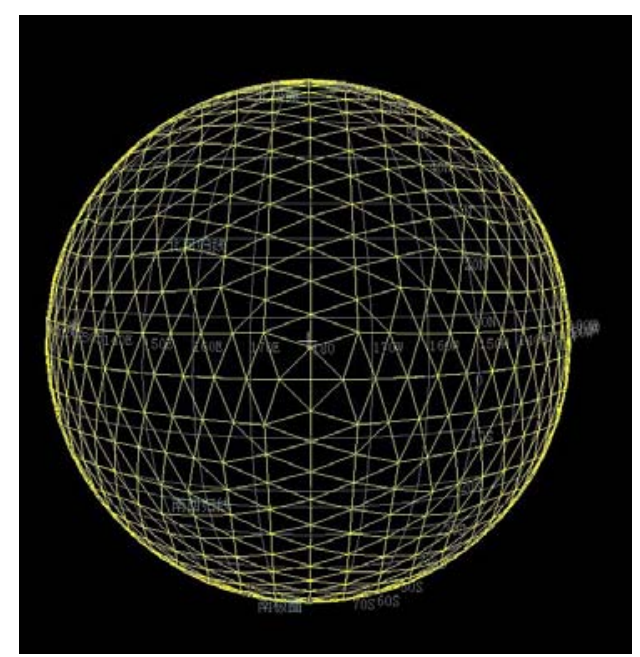

(a)

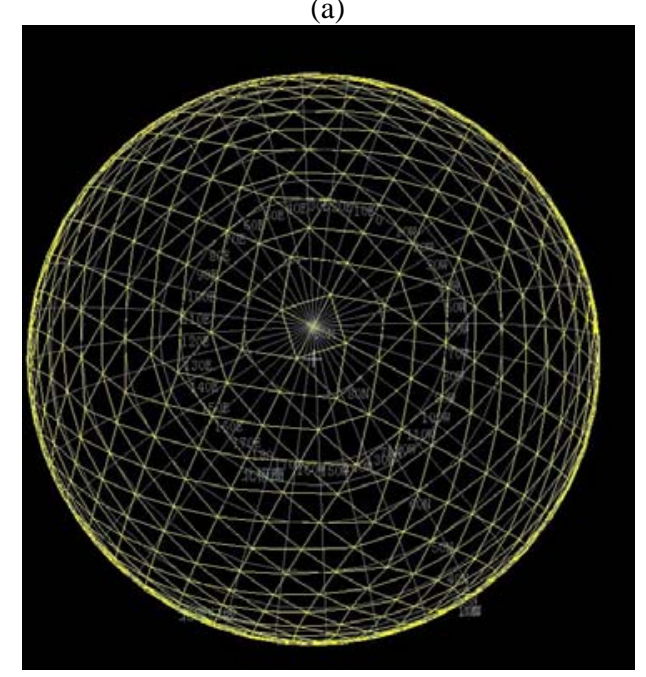

(b)

Figure 8 visualization of Snyder projection grids Grids based on octahedron and Snyder projection provides equal-area and hierarchy characteristic. But it is clean that length and angle distortion is inevitable for equal-area projection. To evaluate uniformity of grid, the ratios of max/min length and angle are used in this paper. After each subdivision, all sphere triangles edge lengths and triangle angles are computed and the ratios of max/min value are acquired. The relations of ratios and subdivision levels are shown as figure 9 and 10. In figure 9, the result indicates that the ratio of max/min edge length is 1.7 , when the subdivision level is equal to 10 . From figure 10, the ratio of max and min angles converges to 3.0.

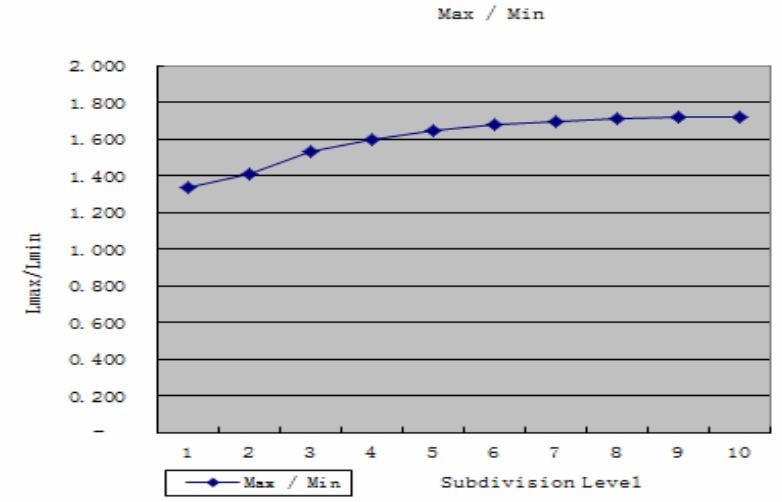

Figure 9 The ratio of max and mix edge length 
SMax $/$ Min

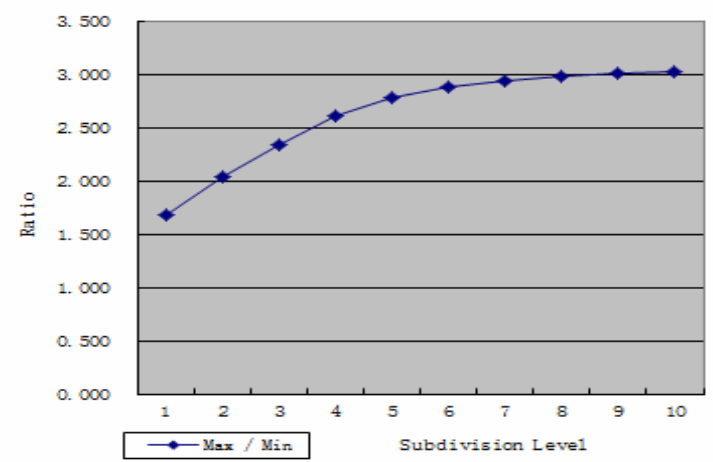

Figure 10 the ratio of max and min triangle angles

\subsection{Discussion}

Most of equal-area subdivision schemes are based on sphere surface. But reference ellipsoid surface is reference basis of most spatial data. What is more, the difference of sphere and ellipsoid cannot be neglected in some spatial analysis, such as spatial sampling, statics analysis etc. Compared with ellipsoid surface, sphere surface is homogenization, smooth, regularity and direction, length, area calculations are easy. Existing GDG subdivision and calculation models on sphere surface is not fit for ellipsoid models. For example, if Snyder projection is used to reference ellipsoid surface, corresponding projection and calculation formulas are invalid. Therefore, hierarchy and equal-area ellipsoid GDG should be discussed.

To acquire practical ellipsoid GDG based on polyhedron, some conditions should be fulfilled. Firstly, there is no gap or overlapping area at matching edges of different faces of polyhedron. Secondly, GDG should provide excellent hierarchy, equal-area characteristic. Thirdly, preferably GDG edge coincides with ellipsoid characteristic line, such as geodesic, longitude and latitude etc[Shi etc 2001, 2006]. Finally, to set up direction, length, area calculation equation is very important. So practical GDG system should be fulfilled four conditions synchronously above. Existing GDG models do not meet conditions above and research works of ellipsoid GDG system should be continued.

\section{CONCLUSION}

For global problem analysis, a data frame is required to store multi-scale spatial data and to be considered as analysis cells in spatial sampling and statistical analysis. To fulfil global statistical analysis requirements, hierarchy and equal-area GDG system is discussed. Octahedron is selected as the base for constructing GDG. Azimuthal equal-area projection and Snyder projection are used for transferring polyhedron surface cells onto sphere surface. Compared with azimuthal equal-area projection method, GDG system with Snyder projection is seamless, hierarchy and equal-area. Length and angle distortion is analysed in the worst cases. The ratios of max, mix length and angle are acquired. Length ratio converges to1.7 and angle ratio converges to 3.0. The result indicates that there exists difference in length and grid angle and the ratios of them are convergent. Unfortunately, existing equal-area and hierarchical GDG system is fit for sphere surface and relative methods cannot be transplanted to ellipsoid surface. So some advices about ellipsoid equal-area GDG are given at last in this paper.

\section{Acknowledgements}

This work has been funded by the National Natural Science Foundation of China (Grant No.41201416 ).

\section{References}

1. Bartholdi J. III and Glodsman P.., 2003. Continuous indexing of hierarchical subdivisions of the globe. International Journal of geographical information science. 15(6): 489-522

2. Bjoke J.T., Grytten J.K., Hager M. and Nilsen S.., 2004. Examination of a constant-area quadrilateral grid in representation of global digital models. International Journal of Geographic Information Science. 18(7): 653-654

3. Bjoke J.T., John K. Grytten J.T. Hager M. and Nilsen S., 2003. A global grid model based on "constant" area quadrilaterals. ScanGIS, 239-250

4. Dutton G., 1999. A hierarchical coordinate system for geoprocessing and cartography. Lecture Notes in Earth Sciences, Springer-Verlag., 230pp

5. Dutton G., 2000. Universal geospatial data exchange via global hierarchical coordinates. The First International Conference on DiscreteGrids'2000. http://www.ncgia.ucsb. edu/globalgrids/ papers(7 Jun. 2012).

6. Goodchild M., Yang S., and Dutton G., 1991. Spatial data representation and basic operations for a triangular hierarchical data structure. NCGIA Report. 91-8, 1-14

7. Gregory M., Kimerling A., White D., and Sahr K., 2008. A comparison of intercell metrics on discrete global grid systems. Computers, Environment and Urban Systems. 32:188-203

8. Harrison E., Madhavi A., Faramarz S., 2011. Optimization of inverse Snyder polyhedral projection. 2011 international conference on cyberworlds. 136-143

9. Harrison E.E., 2012. Equal area spherical subdivision. Master thesis of University of Calgary..1-158

10. Kiester A.R. Sahr K., 2008. Planar and spherical hierarchical multi-resolution cellular automata. Computer, Environment and urban systems. 32: 204-213

11. Kimerling A.J., Sahr K., White D. and Song L., 1999. Comparing geometrical properties of global grids. Cartography And Geographic Information Science, 26(4): 271-288

12. Kolar J., 2004. Representation of the geographic terrain surface using global indexing. Proceeding of 12th International Conference on Geoinformatics. Sweden. 321328

13. Ma T., Zhou C.H., Xie Y.C., Qin B. And Ou Y., 2009. A discrete square global grid system based on the parallels plane projection. International Journal of Geographical Information Science. 23(10): 1297-1313.

14. Ming T, and Zhuang D.F., 2009. The geometrical distortion of discrete global grid in multi-resolution expression. 2009 international conference on environmental science and information application technology. 318-321

15. Ming T, Yuan W. Li S. and Peng G.X., 2011. The expression of discretization line and error analysis in discrete global Grid system. $20113^{\text {rd }}$ international conference on environmental science and information application technology.10: 478-483

16. Leopardi P., 2006. A partition of the unit sphere into regions of equal area and small diameter. Electronic Transactions on Numerical Analysis. 25: 309-327

17. Oldham D. Davies J.H. and Phillips T.N., 2012. Generic polyhedron grid generation for solving partial differential 
equations on spherical surface. Computer \& Geosciences. 39: $11-17$

18. Rosca D., and Plonka G., 2011. Uniform spherical girds via equal area projection from the cube to the sphere. Journal Computer Application Math. 236: 1033-1041

19. Sahr K., White D. and Kimerling. A., 2003. Geodesic discrete global grid systems. Cartography and Geographic Information Science. 30(2): 121-134

20. Sahr K., 2008. Location coding on icosahedral aperture 3 hexagon discrete global grids. Computers and Environment and Urban Systems. 32: 174-187

21. Savric B., Jenny B., Patterson T., Petrovic D. and Hurni L., 2011.A polynomial equation for the natural earth projection. Cartography and Geographic Information Science. 38(4): 363-372.

22. Schneider M., Klein R., 2007. Efficiency and accurate rendering of vector data on virtual landscapes. Journal of WSCG. 15(1-3): 58-65

23. Seong J.C., 2005. Implementation of an equal-are girding method for global-scale image archiving. Phtogrammetric Engineering\& Remote Sensing. 71(5): 623-627

24. Shi Y.M. and Feng Y. 2001. Establishment of another form of geodesic coordinate system on earth ellipsoid(In Chinese). Journal of Tongji University. 29(11):1282-1285

25. Shi Y.M. and Zhu Z.Y., 2006. Computation of area for convex polygon on earth ellipsoid by mean of geodesic coordinate(In Chinese). Journal of Tongji University (natural Science). 34(4): 504-507

26. Song L., Kimerling J., and Sahr K., 2000. Developing an equal area global gird by small circle subdivision. Proceeding of International Conference on Discrete Global Grids. Sanata BarBara, 56-71

27. Snyder J.P., 1992. An equal-area map projection for polyhedral globes. Cartographica: The International Journal for Geographic Information and Geovisualiza-tion. 29(1): 10-21

28. Titz S., Kuhlbrodt T. and Feudel U., 2004. Grid geometry effects on convection in ocean climate models: a conceptual study. Ocean modelling. 7: 165-181

29. Zhao X.S. Cui M.J. Li A. and Zhang M.J., 2009. An adjacent searching algorithm of degenerate quadtree grid on spherical facet(In Chinese). Geomatics and information science of Wuhan University. 34(4): 479-482

30. Yuan W., Zhuang D., and Qiu D., 2010. Equal arc ratio projection and a new spherical triangle quadtree model. International Journal of Geographical Information Science. 64(1): 93-103

31. Vine A. and Zhang X., 2009. Arithmetic and Fourier transform for the PYXIS multi-resolution digital Earth model. International Journal of Digital Earth, 2(1): 59-79

32. White D., 2000. Global grids from recursive diamond subdivisions of the surface of an octahedron or icosahedron. Environmental Monitoring and Assessment. 64(1): 93-103 